\title{
New diagnostic methods aimed at increasing of reliability and safety of the main pipeline transport
}

\author{
Mikhail Sukharev ${ }^{1,2}$, Ksenia Kosova ${ }^{1,2, *}$ \\ ${ }^{1}$ Gubkin Russian State University of Oil and Gas, 119991, Moscow, GSP-1, Leninsky prosp., 65, Russia \\ ${ }^{2}$ LORES Engineering LLC, Moscow obl.,142700, Vidnoe, Zavodskaya ul., 2A, Russia.
}

\begin{abstract}
The paper considers the problems of detection of fluid leaks and calibration of measuring devices in main pipeline systems. On the example of the gas and oil products transport systems we propose new methods for solving these problems. The methods are based on the processing of measurements of standard measuring devices. The efficiency of the methods is verified by numerical calculations by processing real telemetry data.
\end{abstract}

\section{Introduction}

The pipeline transport systems for gas, oil and petroleum products play an extremely important role in the energy supply of the global community. For this reason, increasing the efficiency, safety and reliability of these systems are the most important tasks of their operation.

One way to obtain the desired results in these areas is monitoring the condition of pipeline systems (PS) using control and measurement instrumentation and telemetry systems. The growing level of IT penetration and computerization allows you to collect and process large amounts of information about fluid flow parameters (pressure, flow, temperature, etc.). At present, some information, received from standard measuring devices, is analyzed by specialized software complexes, which contributes to more justified adoption of operational decisions. However, the information potential of the PS is not limited to this.

Detailed data of fluid flow regimes allow us to develop existing and create new methods for increasing the reliability and safety of the main pipeline transport. Such methods are proposed in the present paper; they are based on processing the measurements of a standard measuring instrument and relate to the field of regime diagnostics. They are not expensive to implement and should be particularly attractive to operators.

Let us consider the two problems of increasing the reliability and safety of the operation of gas, oil and product pipelines. It is possible to advance in solving these problems involving new methods of regime diagnostics. The first of them is connected with the detection of leaks and illegal tie-in in the oil and product pipelines, the second - with the adjustment of a set of measuring instruments installed at the gas transmission system (GTS). Despite the fact that each of these tasks relates to a specific technology area, the approaches used to solve them can be developed and used also for other PS.

\section{Detection of leaks and illegal pipelines taps}

Rapid detection of leakages and illegal pipelines taps is one of the priority tasks of ensuring the safe operation of oil and product pipelines. Leakages [1,2] and illegal taps [3] cause serious environmental problems and economic and reputation damage to operator companies.

The problem of the early detection of the leaks and their location in pipeline system has attracted the attention of researchers for more than a century. Beginning with the work of Zhukovsky N.E. (19th century), to identify leakages of oil and petroleum products a wide range of methods was developed. They are based on the registration and investigation of physical processes occurring during the formation of the leak.

Some methods, e.g., $[4,5]$ register front of the pressure wave that appears at the leak occurrence time. Others analyze the acoustic noise accompanying the leak $[6,7]$. For identification of leakages $[8,9]$ also present balance methods: a leak signal is supplied when imbalance flows at the inlet and outlet of the pipeline occurs. In [10-12] for leaks detection the authors employ information about the mathematical models describing the flow regime of the fluid. In the paper [11] a leak detection procedure uses the stability criterion of Lyapunov. The approaches presented in $[10,12]$ are based on comparison operating parameters measurements with simulation results: the difference of the measured and calculated values indicates a leak. To take into account the influence of measurement errors of regime parameters on the results of leak detection methods the publications $[9,13]$ propose the methods of mathematical statistics. To improve effectiveness of the 
leak detection methods in [14] noise in the original data is removed by the wavelet analysis. In the standard [15] for leak detection wave acoustic emission is analyzed. Of course, these approaches do not represent the entire spectrum of the leaks identification methods. Additional information on this topic can be found e.g. in the Handbook [13] or paper [16]. [17] presents a review of pressure-based methods.

Modern information equipment of oil and product pipelines allows us to offer a new method for leaks and illegal taps detection that uses the apparatus of mathematical statistics and the theory of random processes (time series). The method described below belongs to the statistical class, but unlike the known methods [9, 13] does not require the recording of mass conservation and knowledge of the hydraulic model of fluid flow in the pipeline. The proposed method uses only regular pressure measurements in the nodes of the pipeline system and does not require knowledge of the flow rate along the pipeline. The developed procedure makes it possible to detect leaks in both stationary and non-stationary regimes of fluid flow.

\subsection{Technological and mathematical formulation of the problem, method of solution}

Depressurization of the pipeline is accompanied by a sharp drop in pressure. The wave propagates along the pipe. A leak can be detected by monitoring of pressure measured by standard instruments. Similar processes, however, can take place when stopping the pumping unit, opening/closing the line crane, etc. Therefore, the complexity of leak detection is due to the need to distinguish the response of flow parameters to the leakage occurrence and other technological causes.

To identify the leak occurrence time consider the sequence of pressure measurements $P(t)$ at the time points $t,(t=1,2, \ldots)$. The sequence $P(t)$ is a random process with discrete time - a time series*. In general, the process is non-stationary, which is due to technological reasons. At a random time $T$, a sudden pressure drop occurs due to leakage, the probabilistic characteristics of the random process $P(t)$ change. It is necessary to determine as soon as possible the time point when this change occurred.

In the statistics of random processes, the problem of detection whether a change in the probabilistic properties has taken place is called change-point problem, and the time $T$ is the change-point [18]. To make a decision as quickly as possible whether the change-point has happened or not methods of sequential analysis have been developed [18, 19]. We have not a priori information on the distribution of the time $T$ and the distribution of the observed sequence $P(t)$ after the change-point. Therefore, to identify the leakage in the

\footnotetext{
${ }^{*}$ It is possible to consider several time series - pressure measurements near place of leakages, but this is not done in this work.
}

pipeline, it is expedient to use nonparametric methods of sequential analysis $[18,19]$.

The idea behind these methods is that the sequence of observations $\xi(t)$ is used to calculate statistics $y(t)=y_{t}$. It defines the binary decision functions $d\left(y_{t}\right)$. If $d\left(y_{t}\right)=1$, at the time $t$ change has occurred, if $d\left(y_{t}\right)=0$, then change was not.

Nonparametric change-detection methods assume that the observed sequence $\xi(t)$ is stationary. For technological reasons, in identifying leakages of oil and oil products the process $P(t)$ is non-stationary, therefore, before applying methods of sequential analysis it is necessary to transform the process $P(t)$ into a stationary form $\xi(t)$. To do this, receiving a new measurement $P(t+1)$ we consider the prehistory of the process $P(t)$, i.e. values at the time points $t-n, t-n+1, \ldots, t,(n \geq 0)$ when change was not detected. According to the observations $P(t-n), P(t-n+1), \ldots, P(t)$ we find a trend $f(t)$ [20]. Calculating $f(t+1)$ - the predicted value of the process $P(t)$ at the time $t+1$ we find the value $\xi(t+1)=P(t+1)-f(t+1)$. Big modulo value $\xi(t+1)$ indicates a sharp (abrupt) change in pressure the appearance of change.

Thus, the proposed leak identification method sequentially checks incoming pressure measurements for the presence of change. If the incoming value $P(t+1)$ differs significantly from the forecast $f(t+1)$, then it is considered that there is a change, and $t-$ the leak occurrence time. Whether or not the difference $P(t+1)$ from the predicted value $f(t+1)$ is great, the method determines using a non-parametric change-point method.

\subsection{The results of calculations - the processing of actual measurements}

We demonstrate the work of the proposed method using the example of a real product pipeline, at which pressure measurements $P(t)$ are carried out at a frequency of $0.5 \mathrm{~s}$. In the calculations it was assumed that $n=19$, as the nonparametric method of sequential analysis the method of cumulative sums (CUSUM) was used [19].

Statistics CUSUM has the form

$$
y_{t}=\min y_{t-1}+\xi(t), 0, y_{0} \equiv 0, t=1,2, \ldots,
$$

decision function -

$$
d\left(y_{t}\right)=I\left(y_{t}<N\right)
$$

where $N<0$ - the critical value (parameter of the algorithm), $I$ - indicator.

In relations (1), (2) it is assumed that the mathematical expectations of the sequence $\xi(t)$ up to the change point $m$ and after the change point $h+m$ satisfy the inequalities 


$$
m>0, h+m<0 \text {. }
$$

If conditions (3) are fulfilled before the change point $y_{t}=0$, and after the change occurrence the values $y_{t}$ decrease. The leak signal is supplied when $y_{t}$ reaches a critical value $N$. The parameter $N$ is selected by the method of computational experiment on the basis of processing of actual realizations of training time series. In the calculations it was selected $N=-0,01$, in order to satisfy inequalities (3), the value $b=0,025$ that characterizes the maximum measurement error was added to the observed values $\xi(t)$.

The algorithm was tested on the following examples.

Example 1. Fig. 1 shows a graph of the pressure $P(t)$ measured by one of the sensors installed on the product pipeline. The period from the beginning of observation and until the time $t_{1}=8345 \cdot 0,5 \mathrm{~s}$ is the response time. Then the leak occurs, which leads to a sharp drop in pressure.

As a result of the algorithm, the leak was detected at the time $t_{2}=8412 \cdot 0,5 \mathrm{~s}$, i. e. in $33.5 \mathrm{~s}$ after its occurrence. Part of the graph $P(t)$ (Figure 1) on a larger scale is shown in Fig. 2. There is also a graph of the function $f(t)$ (trend) obtained during the operation of the algorithm. Functions $f(t)$ and $P(t)$ are visually indistinguishable. Fig. 3 shows a stationary random process $\xi(t)$ at $t \geq 8000 \cdot 0,5 \mathrm{~s}$.

Example 2. Fig. 4 shows a graph of the wave process, the pressure $P(t)$ from the time $t=58000 \cdot 0,5 \mathrm{~s}$. The actual leak occurrence time $t_{1}=58581 \cdot 0,5 \mathrm{~s}$ in Fig. 4 is marked with a dotted line. The algorithm allowed identifying the leak occurrence time as $t_{2}=58582 \cdot 0,5 \mathrm{~s}$, that is, almost instantaneously, $0.5 \mathrm{~s}$ after its occurrence. In Fig. 4, the time points of leakage occurrence and its recognition are visually indistinguishable.

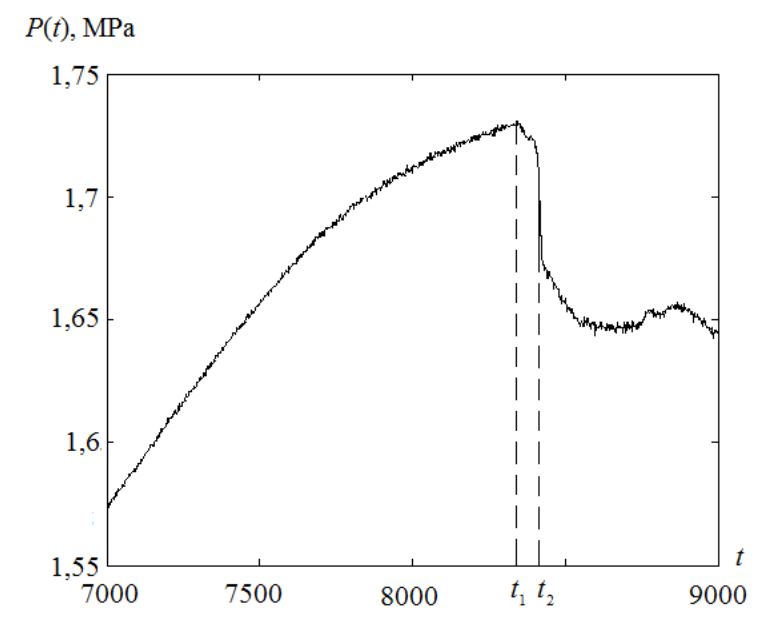

Fig. 1. Example 1. Pressure measurements $P(t)$.

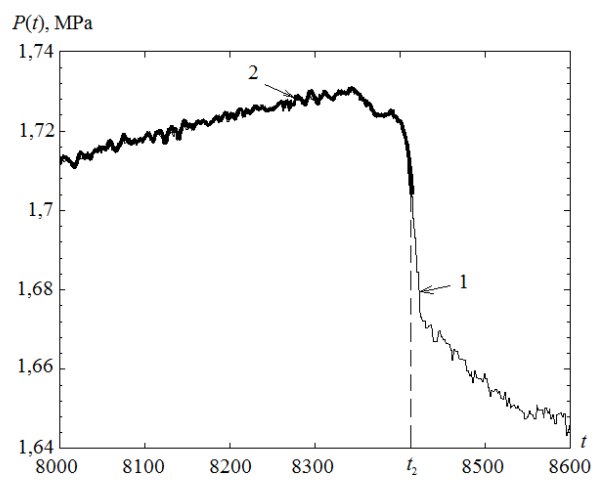

Fig. 2. Example 1. Graphs of functions. $1-P(t), 2-f(t)$.

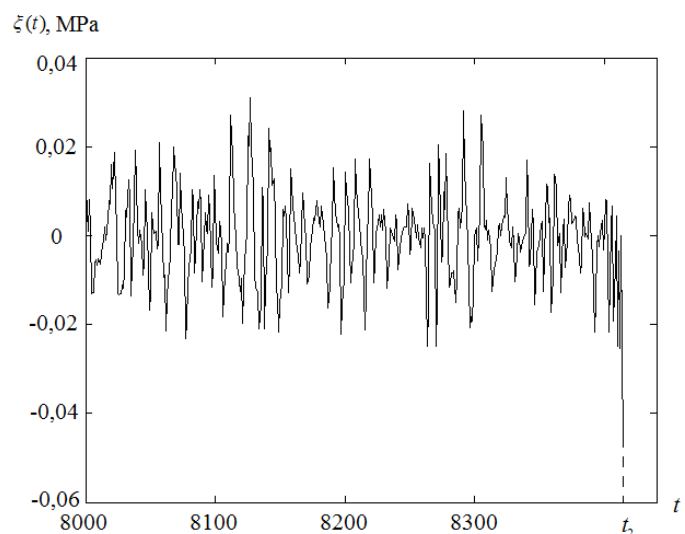

Fig. 3. Example 1. Graph of function $\xi(t)$.

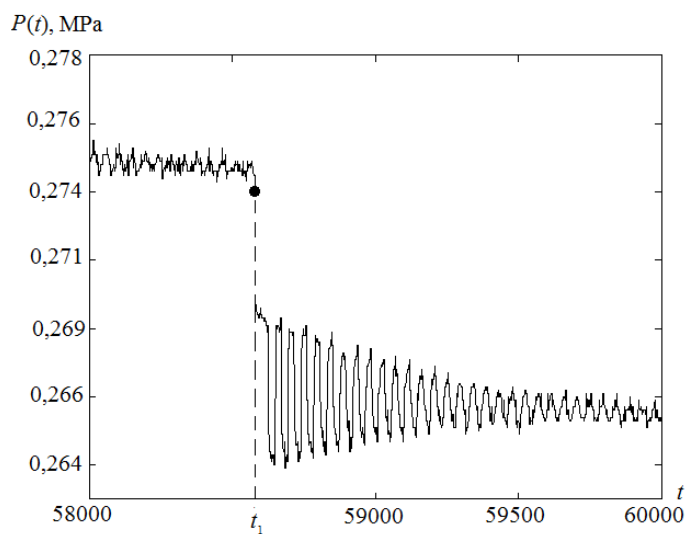

Fig. 4. Example 2. Pressure measurements $P(t)$.

The effectiveness of the method was also checked by examples without leaks.

Example 3. Fig. 5 shows one such case. The wave process that started at the time point $t_{3}=58700 \cdot 0,5 \mathrm{~s}=489,2 \mathrm{~min}$ is a consequence of the pumping unit shutdown (which took place before the time $t_{3}$ ). At the time $t_{4}=65020 \cdot 0,5 \mathrm{~s}=541,8 \mathrm{~min}$ the valve was opened. It would seem that there must be change-points in the same way as in examples 1 and 2 . 
However, the program does not record such time points. This means that there are not false alarms of the method when stopping the pumping unit and opening the valve.

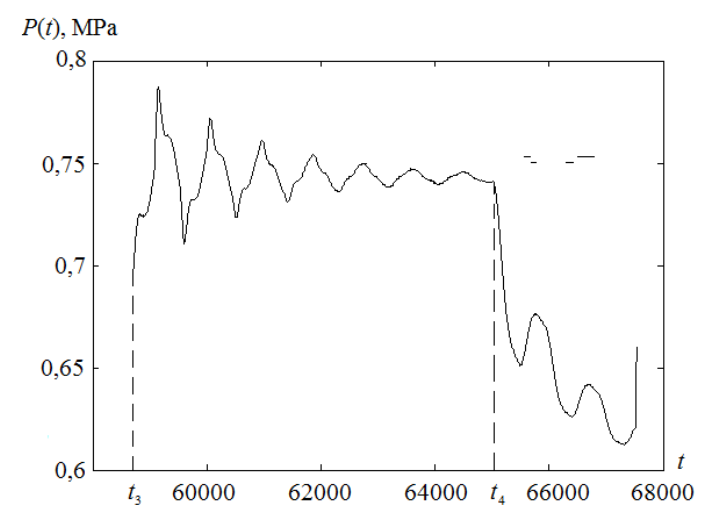

Fig. 5. Example 3. Pressure measurements $P(t)$.

Calculations show that processing of incoming measurements by the method can be performed in real time, a leak is quickly detected after its occurrence. The reactions of the method to leakage occurrence and to disturbances caused by other technological reasons: the valve opening and the shutdown of the pumping unit, differ from each other. Such a positive result is largely due to the fact that before the application of the CUSUM algorithm the trend is removed, that is, the model of the nonstationary random process is transformed into the model of stationary process. The successful choice of the parameter $n$ - the length of the prehistory - allows us to predict adequately the further behavior of the process and prevent false alarms.

Of course, one cannot guarantee a priori that a method can always distinguish a leak from disturbances caused by other causes. However, we recall that the method uses standard measuring instruments and its implementation does not require additional equipment. This determines the expediency of further research and improvement of the proposed apparatus.

\section{Verification of measuring instruments}

Based on the improvement of information support, we offer one more way to improve the safety and reliability of the PS operation. The main source of information about fluid flow regimes is the instrumentation installed on the PS. Ensuring the quality of measurements is a necessary condition for the highly reliable and safe operation of equipment and PS as a whole. Errors in the initial data reduce the confidence level of calculations and can lead to the adoption of incorrect management decisions.

Increasing the quality of the information collected are periodic calibration and verification of measuring instruments. These procedures are an integral part of the maintenance of the PS. Their methodological basis has long been developed; the regulations for their conduct have been approved and have changed little since the adoption, over the course of decades. Qualitative changes in the field of PS computerization allow us to propose new methods of verification and calibration of devices that correspond to the current level of technology. One of these methods is described below. It is intended to correct the measures of the pressure sensors during the operation of the hydraulic system. The effect is achieved through the processing of interconnected measurements of several sensors. In the case of power systems, a similar approach has been developed, in particular, in the works [21-23].

Let us demonstrate the ideological side of the proposed methodology on the example of a gas transportation system (GTS) - a two-line technical corridor of main gas pipelines (Fig. 6). The outer diameters of the pipes are $\mathscr{\varnothing}=1420 \mathrm{~mm}$ (line $I$ ) and $\mathscr{\varnothing}=$ $1220 \mathrm{~mm}$ (line $I I$ ). The length of the route is $176.9 \mathrm{~km}$. There are 9 measuring points on the object; the index $i$ is used for their numbering $i=1, \ldots, 9$ (see Fig. 6). The measuring points are divided the object into 8 pipeline sections. Each section is assigned the number of the initial (in the gas flow direction) point $i=1, \ldots, 8$. The lines are in isolation regime, only the jumpers are open at the beginning and end of the corridor, all the jumpers along the route are closed.

Pressure, temperature and flow measurements were taken over the observation interval of 10 days. Manometers and thermometers are located at each jumper. The flow is measured at the end of the corridor, at the inlet of the compressor station (CS) 2. Fig. 7 and 8 show graphs of pressure measurements at $t \geq 5$ day in the line $I$ (Fig. 7) and in the line $I I$ (Fig. 8). It was found that the temperature exerts a significantly less influence on the results of the calculation than the pressure, so the information on the temperature regime is omitted here.

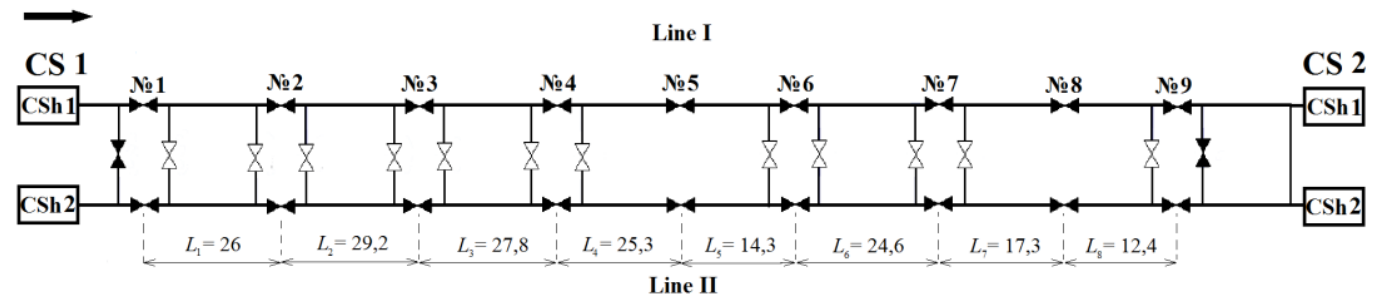

Fig. 6. Schematic diagram of the GTS. Section lengths, $L_{i},(i=1, \ldots, 8) \mathrm{km}$. Unpainted jumpers are closed, painted jumpers are opened. 


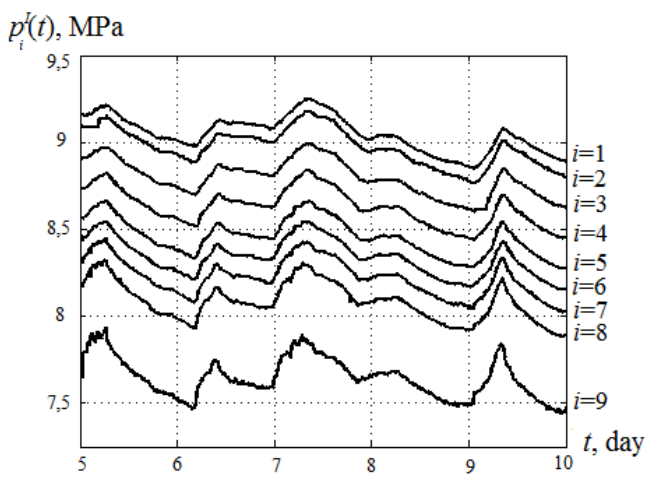

Fig. 7. Graphs of functions $p_{i}^{I}(t), i=1, \ldots, 9$ - pressure measurements in the line $I$.

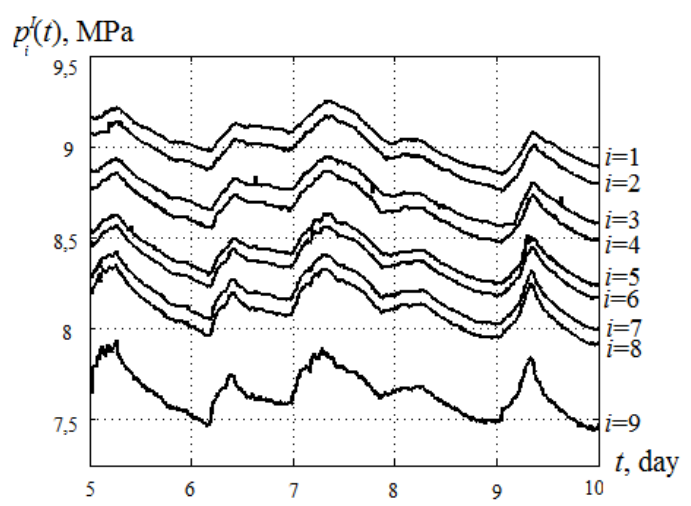

Fig. 8. Graphs of functions $p_{i}^{I I}(t), i=1, \ldots, 9$ - pressure measurements in the line $I I$.

\subsection{Mathematical model}

To describe the interdependence between the parameters of the gas flow, we will use the model of stationary quasi-isothermal flow. The considered corridor is horizontal, therefore the model looks like [24]

$$
p_{s}^{2}-p_{f}^{2}=\Lambda D^{-5} L E^{-2} q^{2},
$$

where $p_{s}, p_{f}$ - pressure values at the beginning and end of the pipeline; $\Lambda$ - coefficient, depending on the characteristics of the pipe, the properties of the gas, the accepted units of measurement; $D$ - internal diameter, $L$ - length of the pipeline; $E$ - coefficient of hydraulic efficiency (used to adapt the results of calculations to actual operating modes); $q$ - commercial gas flow. The temperature is calculated in accordance with Shukhov's formula [24].

The formula (4) is applicable to each section of the corridor. For anyone $i=1, \ldots, 8$, you can write

$$
p_{i}^{2}-p_{i+1}^{2}=\Lambda_{i} D_{i}^{-5} L_{i} E_{i}^{-2} q_{i}^{2} \text {. }
$$

Note that the values $D_{i}$ and $q_{i}$ depend on the line number. For all pipeline sections of the same line they are equal $q_{1}=\ldots=q_{8}=q, \quad D_{1}=\ldots=D_{8}=D$. The differences of the coefficients $\Lambda_{i}$ can be neglected. In addition, there is no reason to consider that the coefficients of hydraulic efficiency $E_{i}$ are different. If this is so, then $E_{1}=\ldots=E_{8}$ and the values $p_{i}^{2}-p_{i+1}^{2}$ differ, mainly because of the lengths of the sections $L_{i}$.

\subsection{Systematic errors in pressure measurements}

Let us analyze the set of functions $p_{i}^{I}(t), p_{i}^{I I}(t), i=1, \ldots, 9$ (Fig. 7, 8). In accordance with the relation (5), the value $p_{i}^{2}-p_{i+1}^{2}$ is proportional to the length of the section $L_{i}$, so, with growth $L_{i}$, the "distance" between the graphs of the functions $p_{i}(t)$ and $p_{i+1}(t)$ in Fig. 7, 8 should increase. It is even visually clear (Fig. 7,8 ) that this condition is not valid. For example, the "distance" between functions $p_{8}(t)$ and $p_{9}(t)$ is more than other "distances" between adjacent graphs $p_{i}(t)$ and $p_{i+1}(t)$ in spite of the fact that the length of section 8 is minimal $L_{8}=12,4$ км. What is the reason for this discrepancy?

We did not find any physical grounds for explaining this effect. During the observation period, hydrate blockage, condensate or sludge deposits were not detected. The corridor was put into operation relatively recently, the flows are high, the gas in the pipeline is well-cleaned and drained. This means that there are no conditions for the appearance of local resistance. In the case of its appearance, the technical services would notice a deviation from the norm (partial failure) and take measures to eliminate it.

The only acceptable explanation for the observed effect is the presence of systematic instrument errors in the pressure measurements. Denote by $\Delta p_{i}$ a systematic error and the measurement result by an asterisk from the top: $p_{i}^{*}=p_{i}+\Delta p_{i}$. Fig. 7,8 show that the most convincing hypotheses about the existence of systematic errors are the following: for the line $I \Delta p_{6}^{I} \neq 0$ and $\Delta p_{7}^{I} \neq 0$, for the line $I I-\Delta p_{2}^{I I} \neq 0, \Delta p_{3}^{I I} \neq 0$ $\Delta p_{4}^{I I} \neq 0, \Delta p_{5}^{I I} \neq 0, \Delta p_{8}^{I I} \neq 0$. In addition, it is useful to evaluate systematic errors $\Delta p_{1}$ and $\Delta p_{9}$ (common for both lines).

We will search the required estimates using the parametric identification algorithm for gas supply systems $[25,26]$. For this purpose, in the parameter identification model, in addition to systematic errors, we introduce the coefficients of hydraulic efficiency $E_{i}^{I}, E_{i}^{I I}, i=1, \ldots, 8$ (which can be considered equal). The identification procedure yields the desired estimates (Table 1). 
The results of calculations presented in the table 1 show that the absolute values of systematic errors are not small. Removing systematic errors from the measurements makes the plot of the pressure change more realistic (Fig. 9).

Thus, the proposed method makes it possible to carry out the procedure of verification and calibration of measuring devices during the operation of the GTS without removing the instruments and using any additional equipment. In calculating operating conditions systematic measurement errors can be properly taken into account reducing the likelihood of incorrect operational decisions. The application of the proposed method is not limited to the example considered. These ideas can be used not only in relation to the technical corridor of gas pipelines, but also to the GTS both treeshaped and looped structure.

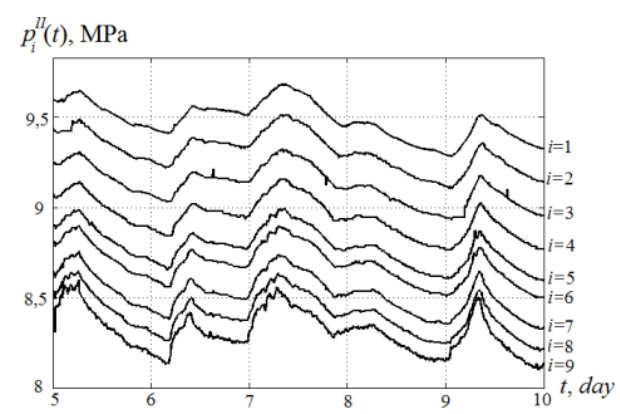

Fig. 9. Graphs of functions $p_{i}^{I I}(t), i=1, \ldots, 9$ after elimination of systematic errors.

\section{Conclusion}

The paper demonstrates the additional possibilities of use of the regime parameters measurements for increasing the reliability and safety of the PS. To this end, we set and solved the following tasks: a) to detect the time of leakage and illegal tapping occurrence in the oil and product pipelines and $b$ ) to verify the measuring instruments installed at the GTS facilities. To solve them we propose new methods of regime diagnostics.

To detect the depressurization time of oil and product pipelines, a technique based on the use of nonparametric change-detection methods was developed. For verification of measuring instruments we presented a method based on algorithms of GTS parametric identification.

The processing of real telemetry data showed the effectiveness of the proposed approaches. Their implementation in production practice will require minimal costs of operators and will increase the safety and reliability of the PS operation.

Table 1. The estimations of the coefficients of hydraulic efficiency and systematic errors.

\begin{tabular}{|c|c|c|c|c|c|c|c|c|c|}
\hline$E_{i}^{I}, E_{i}^{I I}$ & $\Delta p_{1}, \mathrm{MPa}$ & $\Delta p_{2}^{I I}, \mathrm{MPa}$ & $\Delta p_{3}^{I I}, \mathrm{MPa}$ & $\Delta p_{4}^{I I}, \mathrm{MPa}$ & $\Delta p_{5}^{I I}, \mathrm{MPa}$ & $\Delta p_{6}^{I}, \mathrm{MPa}$ & $\Delta p_{7}^{I}, \mathrm{MPa}$ & $\Delta p_{8}^{I I}, \mathrm{MPa}$ & $\Delta p_{9}, \mathrm{MPa}$ \\
\hline 0,9929 & $-0,1032$ & $-0,0171$ & $-0,0439$ & 0,0427 & $-0,0291$ & $-0,0262$ & 0,0283 & 0,0329 & $-0,3334$ \\
\hline
\end{tabular}




\section{References}

1. D. Sharkov. Russia wasting millions of tons of oil from leaking pipes. URL: http://www.newsweek.com /2015/04/17/russia-need-not-drill-arctic-if-it-repairs-oilpipeline-leaks-321372.html

2. It's A Big One': Iowa Pipeline Leaks Nearly 140,000 Gallons Of Diesel. URL: http://www.npr.org/ sections/thetwo-way/2017/01/26/511636325/its-a-bigone-iowa-pipeline-leaks-nearly-140-000-gallons-ofdiesel

3. Mexico's Pemex curbing pipeline thieves with unusable gasoline. URL: http://www.cbc.ca/news/world /mexico-s-pemex-curbing-pipeline-thieves-withunusable-gasoline-1.2960890

4. P. Ostapkowicz. Maint. Reliab. 16(1), 9-16 (2014).

5. S. Siebenaler, E. Tervo, P. Vinh, C. Lewis. IPC 2014. Paper no. IPC2014-33557.

6. J. E. Hough. Oil Gas J. 86(47), 35-41 (1988).

7. B.-W. Yang, M. Recane. U.S. Patent no. 6668619 (2003).

8. H. H. Brons, H. Schaffhaussen. Pipe Line Ind. 5, 50-53; 6, 64-66 (1972).

9. J. Zhang. Pipeline Gas J. 228(2), $42-45$ (2001).

10. J. C. P. Liou, J. Tian. J. Energy Resour. Technol. 117, 243-248 (1995).

11. K. E. Abhulimen, A. A. Susu. Chem. Eng. J. 97, 47-67 (2004).

12. K. F. K. Oyedeko, H. A. Balogun. Int. J. Energ. Tech. Pol. 5 (1), 16-27 (2015).

13. M. Henrie, P. Carpenter, R. E. Nicholas. Pipeline leak detection handbook (Elsiever-Gulf Professional Publishing, United Kingdom, Oxford, 2016).

14. X. Lang; P. Li; Z. Hu; H. Ren; Y. Li. IEEE Access. 5, 8659-8668 (2017).

15. ISO 18081: 2016. (2016).

16. T. R. Sheltami, A. Bala, E. M. Shakshuki. J. Ambient Intell. Humaniz. Comput. 7(3), 347-356 (2016). 17. A. Abdulshaheed, F. Mustapha, A. Ghavamian. Renew. Sust. Energ. Rev. 69, 902-911 (2017).

18. I. V. Nikiforov. Sequential detection of changes in time series properties (Nauka, Moscow, 1983) (in Russian).

19. B. E. Brodsky, B. S. Darkhovsky. Nonparametric methods in change-point problems (Kluwer Academic Publishers, The Netherlands, 1993).

20. M. G. Sukharev, Forecasting methods. (Russian State University of Oil and Gas, Moscow, 2009) (in Russian).

21. J. Zhu, A. Abur. IEEE Trans. Power Syst. 21(2), 586-592 (2006).

22. L. Zhang, A. Abur. IEEE Trans. Power Syst. 28(4), 3916-3923 (2013).

23. I. N. Kolosok, E. S. Korkina, L. A. Gurina. Methodological problems in reliability study of large energy systems. 66, 231-237(2015).

24. M. G. Sukharev, R. V. Samoylov. Analysis and control of stationary and non-stationary regimes of gas transport (Gubkin Russian State University of Oil and
Gas (National Research University), Moscow, 2016) (in Russian).

25. M. G. Sukharev, K. O. Kosova. Autom. Remote Control. 78(5), 882-890 (2017).

26. M. G. Sukharev, K. O. Kosova. Methodological problems in reliability study of large energy systems. 67, 110-119 (2016). 